PROGRESS REPORT NO. 4 to the

i. - DIVISION OF RESEÁRCH

U. S. ATOMIC ENERGY COMMISSION

on

Contract No. AT $(11-1)-1629$

THERMAL MEASUREMENTS ÓN SOLIDS

BELOW $1 \circ \mathrm{K}$

by
Harold Weinstock
Associate Professor of Physics
February, 1970

Department of Physics

Iilinois Institute of Technology

Technology Center

Chicago, Illinois 60616

$\uparrow$ 


\section{DISCLAIMER}

This report was prepared as an account of work sponsored by an agency of the United States Government. Neither the United States Government nor any agency Thereof, nor any of their employees, makes any warranty, express or implied, or assumes any legal liability or responsibility for the accuracy, completeness, or usefulness of any information, apparatus, product, or process disclosed, or represents that its use would not infringe privately owned rights. Reference herein to any specific commercial product, process, or service by trade name, trademark, manufacturer, or otherwise does not necessarily constitute or imply its endorsement, recommendation, or favoring by the United States Government or any agency thereof. The views and opinions of authors expressed herein do not necessarily state or reflect those of the United States Government or any agency thereof. 


\section{DISCLAIMER}

Portions of this document may be illegible in electronic image products. Images are produced from the best available original document. 


\title{
Progress Report No. 4
}

on

\section{Thermal Measurements on Solids Below $1^{\circ} \mathrm{K}$}

\author{
submitted by \\ Harold Weinstock \\ Department of Physics \\ Illinois Institute of Technology
}

This report summarizes the work done on this project from February, 1969 to the present. The participating staff consisted of H. Weinstock (Associate Professor of Physics), C. Chau (Assistant Professor of Physics), R. A. Guenther (Visiting Assistant Professor of Physics), G. Kurz (Research Associate) and G. Jirgal (NSF Summer Faculty Fellow), the following graduate students: C. Ni, R. V. Carlson, S. Lu, M. Sayman, and S. Huang; and undergraduate students: D. Smith, M. Pieczonka, and D. Dzurek.

I. Summary of Research Progress

A - Thermal Conductivity of Cobalt Chloride Thiourea, $\mathrm{CoCl}_{2}\left[\left(\mathrm{NH}_{2}\right)_{2} \mathrm{CS}\right]_{4}$, in Zero and Externally Applied Magnetic Fields

This segment of the research program has been concluded for the most part. A complete account of this forms the basis of the Ph.D. thesis of Chen-Chou $\mathrm{Ni}$ (Ref. 1 and COO-1629-16), copies of which accompany this report. A concise report of the results contained therein has already been submitted for publication (Ref. 2 and C00-1629-14) and a more complete report is now being prepared for submission to the Physical Review.

LEGAL NOTICE

da report was prepared as an account of Covernment sponsored work. Net ther the Untted A. Makes any warranty or representation, expron bebalf of the Commisalon:

racy, completeneas, or usefulnegs of tha tintormesped or implled, whth respect to the accuof any information, opparatus, method, or process disclosed in this report, or that the use prlvately ouned rlghto; or

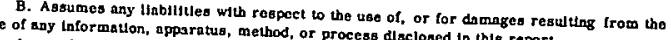
As uzed in the above, "peraon schod, or process dlsclosed In thes report.

ployee or contractor of the "person acung on bebalf of the Commisslon" includes any amsuch employes or contractor of the Commorigsion or of such contractor, to the extent that 
The measurements have been carried out on two specimens, both cut (by a wet string technique) from the same large, solution grown single crystal. One specimen had its long (heat flow) axis in the [0ol] direction, the direction of sublattice magnetization in the antiferromagnetic state. The other specimen had its long (heat flow) axis in the [110] direction, a direction perpendicular to that of the sublattice magnetization. Both specimens were measured over a temperature range of $0.4-18^{\circ} \mathrm{K}$ with the applied magnetic field direction and magnitude as additional parameters.

The results of these measurements indicate the following:

(1) In the paramagnetic state $\left(T>T_{n}=0.92^{\circ} \mathrm{K}\right)$, the cobalt ion forms a simple Kramers doublet. This is seen through the shift in phonon-paramagnetic resonance absorption to higher temperatures with higher applied fields. It is also seen that there is an anisotropy in the g-factor with $g \sim 4$ for the [001] direction and $g \sim 1$ for the[110] direction.

(2) In the vicinity of the Neel temperature, a relatively sharp drop in conductivity (for heat flow in the [00I] direction) is observed and is attributed to scattering of phonons by critical fluctuation of the spin energy density. Furthermore, it is found that this sharp drop is a maximum when the sample is in compression, i.e., supported from below, and is a minimum--disappearing completely--when the sample is under tension, i.e., supported from above. When the sample is supported horizontally, an intermediate effect is observed. In all other 
regions of temperature, the data obtained for the sample in the different positions match within a small experimental error. This behavior is believed to be evidence of a sensitive mechanical coupling to the paramagnetic spin system and shows strikingly a strong mechanical effect on spin alingnment.

(3) In the temperature range below the Neel temperature, the specimen with heat flowing in the [001] direction exhibits an enhanced conductivity, while the specimen with heat flowing in the [110] direction exhibits only a smooth continuation of the $\mathrm{K}_{\mathrm{N}} \mathrm{T}^{3}$ dependence attributed to scattering of phonons by internal boundaries. When an external field of 10 kilogauss is applied to the [001] specimen, its conductivity is reduced and exhibits a $\mathrm{K} \times \mathrm{T}^{3}$ dependence which joins smoothly with data showing a similar dependence above the Néel temperature. Analysis of the zero field 001 data as the sum of two conduction mechanisms yields a $\mathrm{K} \times \mathrm{T}^{3}$ term which exactly (to within experimental error) matches the data obtained in the 10 kilogauss field. Using this analysis and along with the qualitatively different behavior for the [110] direction of heat flow, it has been postulated that the enhancement in conductivity below the Néel is due to magnon conductivity. This postulation has been fortified by qualitative theoretical calculations which indicate that there is sufficient thermal energy available for magnon excitation in the temperature range 
of observation. These calculations were based on a well known

expression ${ }^{3}$ for magnon excitation in the limit $k \rightarrow 0$ for a z-aligned b.c.c. two sublattice antiferromagnet, along with experimentally determined values of $A u$ et al ${ }^{4}$ for the perpendicular and parallel susceptibilities and a critical field value determined in the current work.

(4) Because of the critical scattering phenomenon reported above, it has been possible to trace the phase boundary in the $H$ vs $T$ plane between the paramagnetic and antiferromagnetic states. Furthermore, there appears to be a sharp drop in thermal conductivity as one increases the field at a constant temperature well below the Neel temperature. This drop is considered to be indicative of a transition from the antiferromagnetic to the spin-flop phase where magnon conductivity is destroyed and has yielded information on this phase boundary which is consistent with similar behavior in other antiferromagnetic systems.

(5) As the magnetic field within the spin-flop region is increased, the conductivity also increases (with temperature held constant). It is speculated that this increase in conductivity is due to the onset of weak ferromagnetic magnon conduction. This speculation must, however, be backed up by susceptibility measurements in a magnetic field at these temperatures for confirmation.

B - Phonon Resonance Scattering in CdS Containing $\mathrm{Co}^{2+}$ The thermal conductivity of CaS doped with 500ppm of $\mathrm{Co}^{2+}$ has been measured from $0.35^{\circ}-4.0^{\circ} \mathrm{K}$ as function of magnetic field 
up to $20 \mathrm{kG}$. A typical conductivity dip appears in all conductivity versus temperature curves as show in Fig. I. The position of the dip shifts to higher temperature with increasing magnetic field. The results are qualitatively in agreement with a phonon resonance scattering process according to Morigaki's $s^{5}$ measured magnetic level scheme of $\mathrm{Co}^{2+}$ in $\mathrm{CdS}$. Because there is a $1.33 \mathrm{~cm}^{-1}$ internal field splitting of the $J=1 / 2$ and $J=3 / 2$ states, a phonon resonance scattering should occur in the zero external field conductivity curve also. This effect is demonstrated in the analysis of the current work.

A Debye thermal conductivity integral has been used for this analysis, with a combined relaxation rate which includes the phonon scattering processes by external boundaries, isotopes, simple point defects of the Rayleigh type and resonances. In the temperature range of this work, the normal and umklapp three phonon processes are not important, and hence they have been neglected in the analysis. The strength of the external boundaries, isotope and simple point defect scattering processes have been calculated according to Klemens' expressions ${ }^{6}$. As for the resonances, a general phonon resonant relaxation rate derived by Klein ${ }^{7}$ has been applied.

In the absence of magnetic transitions, the $\mathrm{Co}^{2+}$ ion will scatter phonons mainly due to the mass difference with its host $\mathrm{Cd}^{2+}$. The resulting conductivity curve based on the theory for 
mass defect scattering is shown in Fig. 2 (curve A), and it is seen that it differs from the experimental curve both in magnitude and shape:

The simplest situation for phonon resonant scattering by the $\mathrm{Co}^{2+}$ magnetic levels is in the zero field case, where there is only one distinct transition from the $J=1 / 2$ to the $J=3 / 2$ state. By adjusting the transition linewidth in the resonant expression added to the mass defect scattering term, curve $B$ in Fig. 2 was obtained, in good agreement with the zero field experimental curve. The deduced linewidth in this case is $4 \times 10^{-4} \mathrm{~cm}^{-1}$, two orders of magnitude larger than one would expect from Orbach's one phonon transition spin-lattice relaxation ${ }^{8}$. However, with $500 \mathrm{ppm}$ of $\mathrm{Co}^{2+}$, the dipole-dipole interaction should be important. With Kittel and Abrahams' expression 9 for dipoledipole interaction, a linewidth similar to the deduced value was obtained. Other effects which could contribute to the linewidth are inhomogeneous broadening due to dislocations, and random electric fields due to the CdS crystal lacking inversion symmetry and possible departure from stoichiometry.

In the presence of a magnetic field, there are six allowed transitions. Morigaki's electron spin resonance experiment showed that the linewidth of the intra-doublet transition is small, and thus this transition should have no effect on the thermal conductivity. The conductivity curves with external fields have been fitted by adjusting the linewidths of the four 
extra-doublet transitions. However, it must be realized that these deduced linewidths are somewhat uncertain because of the large number of parameters used in the curve fitting. It is considered desirable therefore, to perform spin-lattice relaxation experiments on the specimen crystal to verify these deduced linewidths.

C - Magnetic Field Dependence of the Basal Plane Thermal Conductivity of Pyrolytic Graphite

The basal plane thermal conductivity of pyrolytic graphite has been measured from $0.35^{\circ}-4.0^{\circ} \mathrm{K}$ in several external magnetic fields along the direction of the heat flow. The conductivity curves show strong field dependence which has been interpreted as due to a large electronic contribution to the heat conduction. This finding is in agreement with the work of Holland ${ }^{10}$ in which the magnetic fields were perpendicular to the direction of the heat flow. Using Kelly's ${ }^{11}$ theoretical calculation, the crystallite size of our sample has been estimated to be on the order of two microns. In the work reported in Ref. 10, the electronic component of the thermal conductivity was gradually quenched with increasing external magnetic field and complete quenching occurred when the external magnetic field reached $3.8 \mathrm{kG}$. However, the current investigation shows substantial change between the $H=4 \mathrm{kG}$ and $\mathrm{H}=16 \mathrm{kG}$ curves. This result may be explained in the following way. Electrons carry heat along the direction of heat flow in the absence of an external magnetic field. But in the presence of fields in the same direction as the heat flow, 
electrons circle about the magnetic field lines as well, and when the radii are larger than the above deduced crystallite size, the electrons travel transversely to the direction of the heat flow, colliding with one side and then the other side of the crystallite boundary. In this case, the heat conduction of the electrons is quenched. Additionally, the cyclotron frequency is proportional to the external magnetic field. Therefore, the electron-phonon scattering rate will increase with increasing external field and the phonon heat conduction should decrease as observed.

According to the above explanation, the curves obtained for various applied magnetic fields could supply information on the strength of the electron-phonon interaction. After substracting out the electronic component, the lattice thermal conductivity of the zero field curve will be analyzed. under the frame-work outlined by Kelly ${ }^{11}$. The information gained here in this study will be used ultimately to provide a foundation for studying defects produced in fast neutron irradiated pyrolytic graphite at low temperatures, a study of considerable practical importance.

D - Thermal Conductivity of Radiation Damaged MgO This work has only just begun. Preliminary measurements have been made on "pure" crystal specimens kindly supplied by Dr. W. A. Sibley of Oak Ridge National Laboratory. Along with these specimens came the information that while relatively pure, these crystals contained bubbles of about 0.5 micron or 
or smaller. The thermal conductivity results indeed support this information.

While a $K \propto \mathrm{T}^{3}$ dependence is seen for $1^{\circ} \mathrm{K}<\mathrm{T}<4.2^{\circ} \mathrm{K}$, the magnitude of the conductivity is a factor of almost 30 below that predicted by simple Debye theory and the Casimir length of $4.3 \mathrm{~mm}$ for the specimen under test. The thermal conductivity is also 5 to 10 times lower than the values reported by others in even more impure samples ${ }^{12,13}$. Additionally in the current work, there is an approximate $\mathrm{K} \propto \mathrm{T}^{2}$ dependence in the range $0.4<\mathrm{T}<1.0^{\circ} \mathrm{K}$. The overall appearance of this data is a relativately sharp change in conductivity in the region of $1 \% \mathrm{~K}$. At that temperature the wavelength of the most numerous phonons is calculated to be $2500 \AA$, i.e., in the same order of magnitude as the diameter of the bubbles. It appears then that the reduced conductivity is a form of geometrical scattering resonance observed in earlier work on radiation damaged $\mathrm{KCl}^{14,15}$. An extensive effort is now. being made to obtain other $\mathrm{MgO}$ crystal specimens both from C. T. Butler of Oak Ridge National Laboratory who has ones of purer quality with 5 to 10 micron bubbles, and from all available commercial sources. When all possible specimens have been obtained, then the radiation damage studies will be made, primarily using neutron irradiation. $E$ - Nuclear Specific Heat of $\mathrm{UO}_{2} \mathrm{Rb}\left(\mathrm{NO}_{3}\right)_{3}$

This work is being carried out at the suggestion and with with the advice of Dr. J.W. T. Dabbs of Oak Ridge National Laboratory. The measurements are being made in the range 
$0.45 \mathrm{~T} 4.2^{\circ} \mathrm{K}$ on two powdered specimens of the above named uranium salt, one specimen containing normal uranium-mostly ${ }^{238} \mathrm{U}$, and the other containing a highly enriched concentration of $235 \mathrm{U}$.

Results have just been obtained on the enriched $235 \mathrm{U}$ specimen and evidence of what appears to be the onset of a nuclear Schottky anomaly has been seen. These results are now being analysed by a least-squares computer fit for a heat capacity temperature dependence which includes a term in $1 / \mathrm{T}^{2}$ for the dependence of the Schottky anomaly tail. Meanwhile, preparations are being made to carry out measurements on the normal ${ }^{238} \mathrm{U}$ specimen of $\mathrm{UO}_{2} \mathrm{Rb}\left(\mathrm{NO}_{3}\right)_{3}$ which is about five times more massive than the enriched salt. Thus, a much more reliable result will be obtained for the magnitude of the "background" lattice specific heat and one will not have to rely entirely on the computer analysis for this term and will in a sense have an independent check of this analysis. The $238_{\mathrm{U}}$ nuclei, of course, are not expected to exhibit the Schottky anomaly. It is believed that the anomaly is related to electric quadrupole splitting of the ${ }^{235} \mathrm{U}$ nucleus; and that the measure of this splitting obtained may provide some corroboration to a theory which predicts a doubly peaked nuclear potential barrier with a shallow isomeric minimum between peaks.

\section{F - Thermal Conductivity of Superconducting Alloys}

Some preliminary work has been done on the conductivity of commercial superconducting alloy wires (of niobium-tin, niobium- 
zirconium and niobium-titanium) which are used in the construction of superconducting magnets. Unfortunately, this work has been temporarily suspended due to a personal tragedy in the family of the student embarking on this study. It is anticipated that this investigation will be resumed, along with those of La-Ga-In superconducting alloys, when additional experienced personnel are available during summer 1970. 


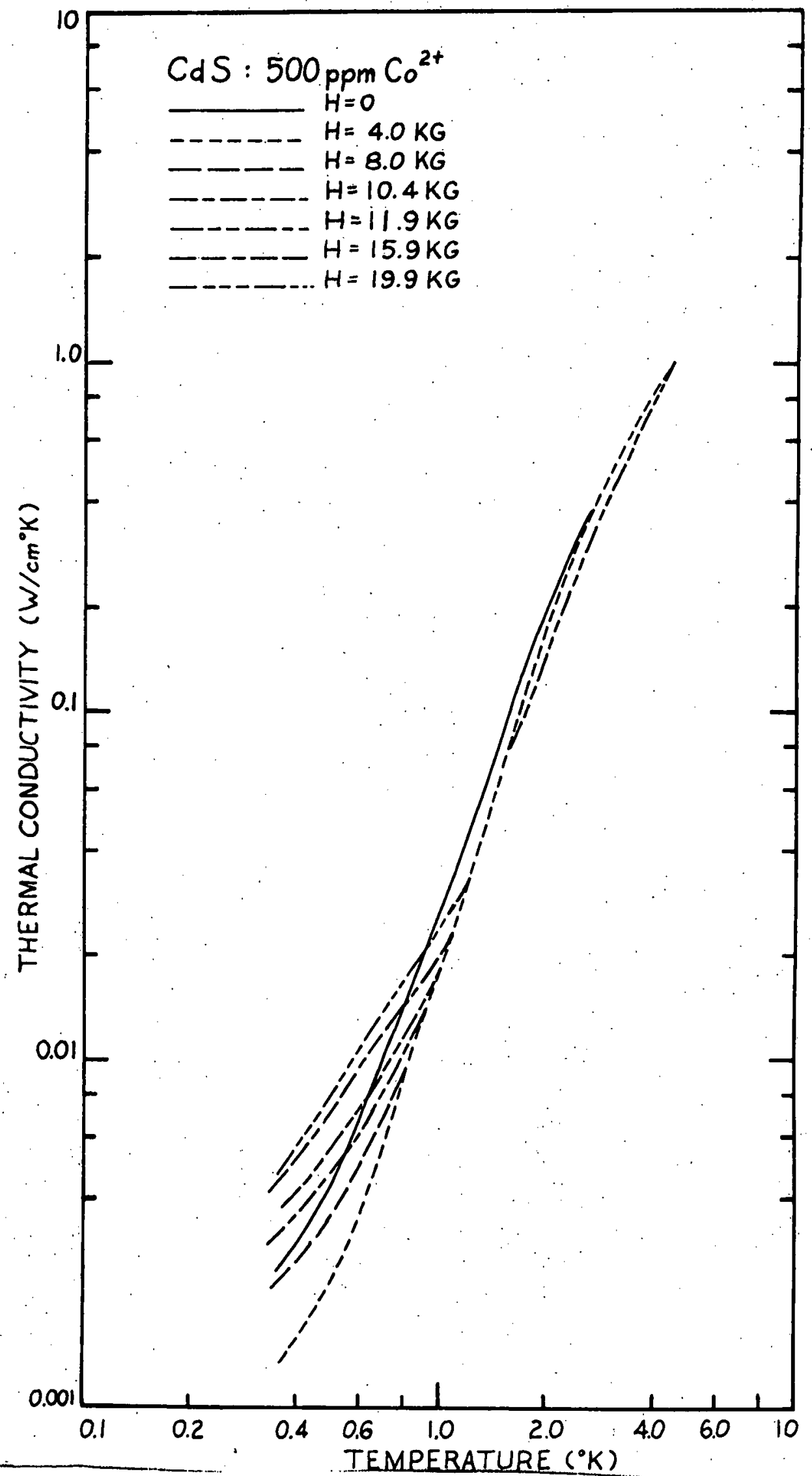

Fig. I Magnetic Field Dependence of Thermal Conductivity of CdS:Co ${ }^{2+}$ 


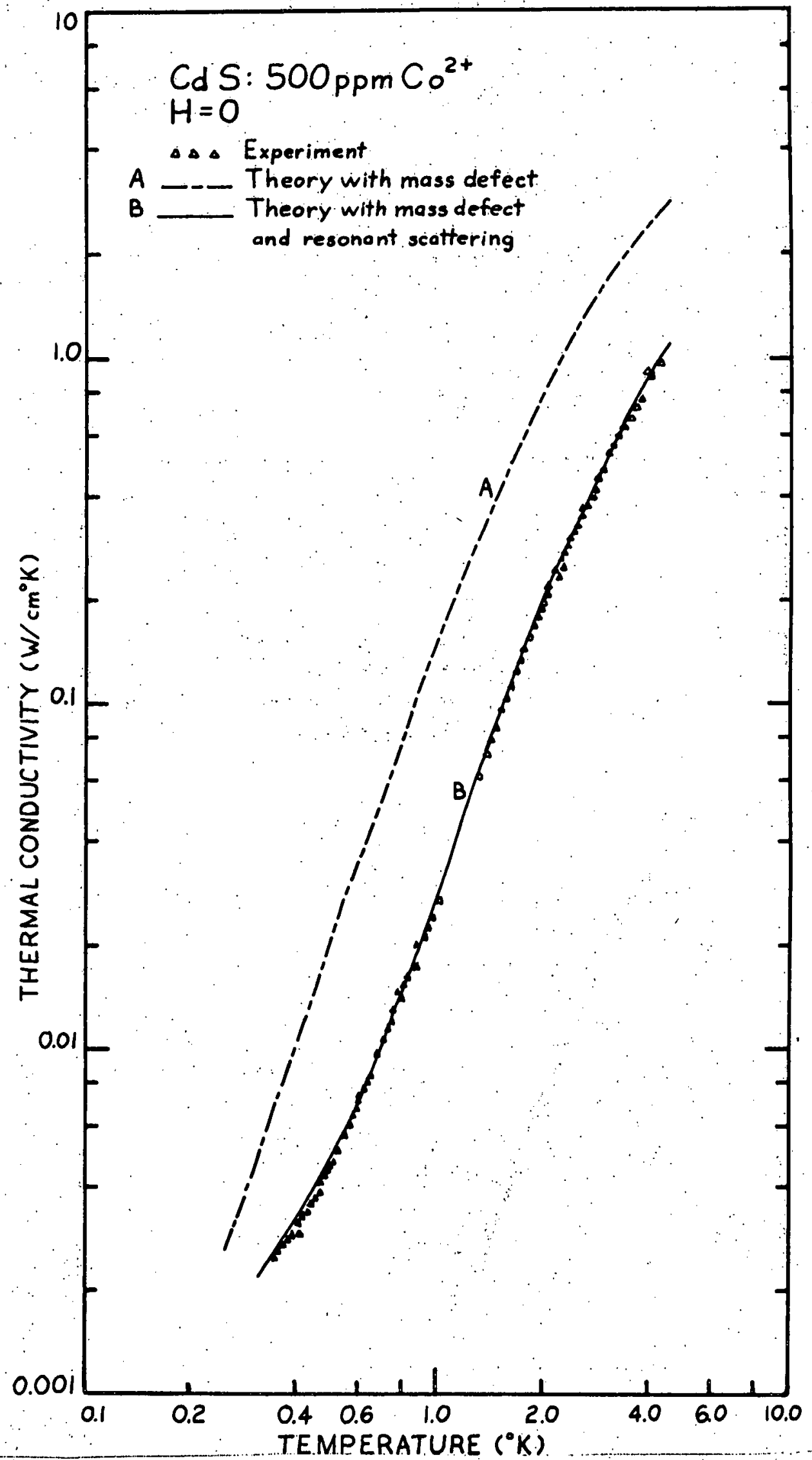

Fig. 2. Experiment and Theory of Zero Field Thermal Conductivity of CdS:Co ${ }^{2+}$ 
1. C. Ni, Ph.D. Thesis; I.I.T., 1970 (unpublished); AEC Document C00-1629-16

2. $\mathrm{C} . \mathrm{Ni}$ and H. Weinstock (submitted to Physica)

3. D. H. Martin, Magnetism in Solids (The MIT Press, 1967)

4. R. Au, J. A. Cowen, R. D. Spence and H. van Till, Low Temp. Phys.-IT9, edited by J. G. Dount et al (Plenum Press, 1965), p. 877

5. K. Morigaki, J. Phys. Soc. Japan 19, 2064 (1964)

6. P. G. Klemens, in Solid State Physics, edited by F. Seitz and D. Turnbull (Academic Press, Inc., New York, 1.958) ?,

7. M. V. Klein, Phys. Rev. 186, 839 (1969)

8. R. Orbach, Proc. Roy. Soc. (London) A264, 458 (1961)

9. C. Kittel and E. Abrahams, Phys. Rev. 90, 238 (1953)

10. M. G. Holland, C. A. Klein and W. D. Straub; J. Phys. Chem. Solids 27,903 (1966)

11. B. T. Kelly, Carbon 6, 71 (1968) and Carbon 6, 485 (1968)

12. G. A. Slack, Phys. Rev. 126, 427 (1962)

13. G. T. Fox, M. W. Wolfmeyer, G. R. Dillinger and D. L. Huber, Phys. Rev. 165,898 (1968)

14. R. A. Guenther, Ph.D. Thesis, I.I.T. 1969 (unpublished); AEC Document COO-1629-11

15. E. Sonder and D. Walton, Phys. Letters 25A, 222 (1967) 
II. Graduate Student Progress

Raymond A. Guenther, who had been awarded a Ph.D. degree in January, 1969 after completing thesis research under the current project, continued his association with the laboratory through August, 1969. At that time he left to assume the position of Associate Professor of Physics at the University of Nebraska at Omaha, where he is currently planning the construction of a small scale low temperature-solid state laboratory.

Chen-Chou Ni recently--January, 1970-- has been awarded a Ph.D. degree after completion of thesis research under the current project. His thesis entitled "Magnetic Field Effects on the Thermal Conductivity of $\mathrm{Co}\left[\left(\mathrm{NH}_{2}\right)_{2} \mathrm{CS}\right]_{4} \cdot \mathrm{Cl}_{2}$ " has been designated AEC Document C00-1629-16 and accompanies this report. He has just recently begun employment as a research scientist at the Naval Research Laboratory near Washington, D.C.

The next most senior student and holder of an NDEA Fellowship, Richard V. Carlson, was progressing quite well both scholastically and in his research activities when the sudden death of his father made it necessary for him to return to the Minneapolis, Minnesota area to take up residence with his remaining family. He has, however, been awarded a fellowship at the University of Minnesota where he is continuing research (started at IIT) in the field of superconductivity under Professor A. Goldman.

A student who joined this project only last fall, Miss S. Lu, has made remarkable progress in her work on the magnetic field dependence of the basal plane thermal conductivity of pyrolytic 
graphite. It is expected that this work will form the basis of an M.S. thesis toward the award of a degree either in June, 1970 or January, 1971.

The remaining graduate students currently employed, M. Sayman and S. Huang, have only just begun their laboratory work. At this stage it is difficult to predict their future progress. For the present, they are both learning basic techniques and assisting in the research activities directed by the more senior members of the laboratory staff.

III. Progress in the Development of Laboratory Facilities As has been the case now for about two years, the original laboratory cryogenic station for attainment of temperatures down to $0.3^{\circ} \mathrm{K}$ is in use on virtually an around-the-clock basis. This high level of activity is facilitated by the fact that any one of three $3_{\text {He cryostats, two for thermal conductivity and one for heat capacity }}$ measurements, may be and are being used interchangably in the station.

A second cryogenic station utilizing helium dilution refrigeration is in the final stages of construction, and while not yet available for use, it is expected to be so by the summer of 1970, if not sooner. This station will incorporate a high field ( $>50 \mathrm{kG}$ ) super-. conducting magnet which has been received only recently and is now undergoing testing. When fully operational, there is good reason to believe that this new wider range cryogenic facility will receive the same extended use as has been given to its predecessor, while at the same time a high level of activity will continue on the older system. 